\title{
Tagungsbericht Herbsttagung der Arbeitsgemeinschaft Knochentumoren 2018
}

Die 91. Tagung der AG Knochentumoren fand vom 19. bis 20. Oktober 2018 wieder in München in den Räumen des Instituts für Pathologie der TU München statt. Gastgeber waren Prof. Dr. Wilko Weichert zusammen mit seinem Team und Frau Katja Specht als lokale Veranstalterin. Sie sorgten auch diesmal für einen reibungslosen Tagungsablauf, so dass sich die Teilnehmer des Arbeitstreffens komplett auf die ad-hoc Fälle, wissenschaftlichen Vorträge und Falldiskussionen konzentrieren konnten. Die wie immer äußerst angenehme Atmosphäre trug ein Übriges dazu bei, dass auch diese Tagung allen Mitgliedern und den zahlreichen Gästen in bester Erinnerung bleiben wird.

Der eigentlichen Arbeitstagung vorgeschaltet fand am Freitagvormittag von 8:45 bis 13:00 Uhr eine von der AG Knochentumoren und der Deutschen Gesellschaft für muskuloskelettale Radiologie (DGMSR) gemeinsam organisiert Fortbildungsveranstaltung zum Thema : „Knochentumoren: Pathologie und Radiologie" statt, die auch für Nichtmitglieder beider Gesellschaften offen war. Ihr diesmaliger Schwerpunkt lag (neben Referaten zur Grundlagen der radiologischen und histopathologischen Diagnostik von Knochentumoren) auf der Darstellung gutartiger und bösartiger Knorpeltumoren.

Die Tagung begann mit der Vorstandssitzung (15:00 Uhr bis 16:00 Uhr) und wurde mit der Präsentation von ad-hoc Fällen fortgesetzt (16:00 Uhr bis 18:00 Uhr). Insgesamt wurden 5 Fälle vorgestellt und diskutiert. Diese Fälle, die kurzfristig über das Sekretariat der AGKT (sekretariat@agkt.org) angemeldet oder spontan eingebracht werden können, beinhalten überwiegend aktuelle diagnostische oder therapeutische Probleme, zu denen Expertenmeinungen aus dem Kreis der AGKT erbeten sind.

Diskutiert wurden ein Fall einer 29-jährigen Frau mit einem wenig matrixbildenden, des- halb konventionell-histologisch schwer zu erkennendem epitheloiden Osteosarkom der Fibula, dass außerdem immunhistochemisch eine ausgeprägte aberrierende Zytokeratinexpression zeigte, welche primär an die Metastase eines Karzinoms denken ließ.

Ein weiterer Fall betraf einen 46-jährigen Patienten, bei dem seit längerer Zeit ein Tumor im rechten Unterschenkel bekannt war, der bisher als „Gefäßtumor“ eingeordnet wurde. Dieser Fall soll nach Vorlage der gesamten - bisher inkonklusiven - Histologie erneut vorgestellt werden. Gleiches gilt für den dritten Fall (60-jähriger Mann mit einem osteodestruktiven, spindelzelligen Tumor der distalen Femurdiaphyse).

Beim vierten Fall handelte es sich um einen 16-jährigen Jungen mit einem Beckentumor (Verdacht auf mesenchymales Chondrosarkom). Auch hier soll ein Verlaufsbericht nach Vorlage weiterer Untersuchungen folgen.

Als Abschluss dieses Sitzungsteils wurde über den Verlauf eines Falles berichtet, der an der letzten Falldiskussion lange erörtert wurde, ohne dass ein Konsens über die Diagnose zustande kam (aneurysmatische Knochenzyste vs. teleangiektatisches Osteosarkom). Zunächst wurde aufgrund der nicht eindeutigen Diagnose auf eine radikale Chirurgie und Chemotherapie verzichtet. Leider erwies es sich im Verlauf, dass die Osteosarkom-Diagnose zutreffend war, so dass eine Re-Operation und Chemotherapie angeschlossen wurde. Zwischenzeitlich verstarb die Patientin jedoch an Therapiekomplikationen.

An die Diskussion der ad-hoc Fälle schlossen sich (18:15 Uhr bis 19:00 Uhr) die wissenschaftliche Vorträgen an, welche im Anschluss diskutiert wurden. Hauptthema war die Klassifikation vaskulärer Läsionen, die in einem gemeinsamen Vortrag aus der Sicht von Pathologie und Genetik vorgestellt wurde.
Vaskuläre Läsionen des Skeletts: Pathologie und allgemeine genetische Aspekte Elisabeth Bruder ${ }^{1}$ und Dorothea Wand ${ }^{2}$ : Institut für Medizinische Genetik ${ }^{2}$ und $\mathrm{Pa}$ thologie ${ }^{1}$, Universitätsspital Basel Schönbeinstrasse 40, 4031 Basel, Schweiz (Die Kurzfassung des Vortrages ist im Anschluss an den Tagungsbericht zu finden: S. $91 \mathrm{ff})$

\section{Mitgliederversammlung}

Der Samstag begann mit der nichtöffentlichen Mitgliederversammlung (8:35 Uhr bis 9:15 Uhr). Vor Eintritt in die eigentliche Tagesordnung gedachten die Teilnehmer ihres im November 2018 verstorbenen Mitgliedes Prof. Dr. Claus-Peter Adler aus Freiburg, der seit der 4. Sitzung 1974 Mitglied der AGKT war, wesentlich an den Diskussionen innerhalb der AGKT teilgenommen hatte und als Osteopathologe weit über den deutschsprachigen Raum hinaus bekannt war. Den Nachruf hielt Prof. Dr. Markus Uhl, sein langjähriger radiologischer Wegbegleiter.

Anschließend erfolgten satzungsgemäß (Ablauf der Amtszeit) die Neuwahlen des Kassenwarts und des Geschäftsführers. Prof. Hillmann (Kassenwart) und Prof. Jundt (Geschäftsführer) erklärten sich erneut zur Kandidatur bereit und wurde in ihren Ämtern ohne Gegenstimmen bestätigt.

Außerdem wurde ohne Gegenstimmen beschlossen, Herrn Dr. Anusch Sufi-Sivach, leitender Oberarzt und Leiter des Schwerpunktes Tumororthopädie an der Schön-Klinik, Hamburg-Eilbek, als neues Mitglied in die AGKT aufzunehmen.

\section{Wissenschaftliche Falldiskussion}

Als Hauptteil des Samstagvormittags folgte dann ab 9:30 Uhr die wissenschaftliche Fall- 
diskussion vorher eingereichter Fälle, deren klinische Informationen, Radiologie und eingescannten Schnittpräparate den Mitgliedern und Gästen vorab im Internet via iPathnetwork.com zur Einsichtnahme und Beurteilung zur Verfügung standen (Moderation. Prof. Dr. Elisabeth Bruder, Basel).

Insgesamt wurden 9 Fälle diskutiert. Das Altersspektrum reichte von 4 bis 67 Jahren. 6 Fälle traten bei Männern und 3 Fälle bei Frauen auf. Betroffen waren die langen Röhrenknochen (6×), das Sacrum (1×), der Fuß (1×) sowie der Radius (1x). Eine Läsion (pseudomyogenes Hämangioendotheliom) trat multifokal im Knochen und den Weichteilen auf. Bei einem Fall eines 4-jährigen Mädchens handelte es sich um die sehr seltene progressive ossäre Hyperplasie, eine genetisch bedingte, mit ektopen Ossifikationen einhergehende Erkrankung, welche auf einer hereditären bzw. einer Neumutation im Exon 1-13 des GNAS- Gens beruht und zum Formenkreis des Pseudohypoparathyreoidismus gerechnet wird. 6 Fälle betrafen maligne Tumoren, die zum Teil eine
Dedifferenzierung und somit den Übergang von low-grade zu high-grade Tumoren zeigten (zwei Osteosarkome, ein Chondrosarkom). Ein diaphysärer, vorwiegend epithelial aufgebauter Tumor wurde bei Abwesenheit eines anderen Primärtumors zunächst als sogenanntes Adamantinom der langen Röhrenknochen eingestuft.

Als weitere Besonderheit wurde eine praktisch Riesenzell-freie, immunhistochemisch und molekularpathologisch aber eindeutig als Riesenzelltumor charakterisierbare Läsion präsentiert, deren mononukleäre Zellen (die eigentliche Tumorzellkomponente, welche normalerweise die namengebenden Riesenzellen sekundär via Rank-RanklInteraktion „anlockt“) die für Riesenzelltumoren pathognomonische H3F3A-Mutation aufwiesen, welche nur in den mononukleären Zellen, nicht aber in den Riesenzellen zu finden ist.

Am Ende der Falldiskussion wurden Frau PD Dr. Sylvia Hoeller, Frau Dipl. med. Dorothea Wand, Prof. Dr. Wolfgang Hartman, Prof.
Dr. Rainer Erlemann und Prof Dr. Jürgen Freyschmidt aus Basel, Münster, Duisburg und Bremen für die Darstellung und Diskussion Ihres Falles 1097 einer („Progressive ossäre Hyperplasie“) der Preis für die beste Fallpräsentation zuerkannt.

Die Tagung wurde gegen 13:30 Uhr mit einem nochmaligen Dank an das Münchener Team für die exzellente Organisation der Veranstaltung und die hervorragende Betreuung beendet.

\section{Zukünftige Tagungen}

Die Frühjahrstagung 2019 findet auf Einladung von Prof. Dr. Sven Seiwerth vom 24. bis 25. Mai in Zagreb statt. Die Herbsttagung wird wie üblich in München (18. und 19. Oktober 2019) stattfinden

Falls Interesse besteht, als Gast an den Tagungen der AG Knochentumoren teilzunehmen, können weitere Informationen über das Sekretariat der AGKT in Basel abgerufen werden (sekretariat@agkt.org). 